1 De Groot LJ, Kaplan EL, McCormick M, Straus FH. Natural history, treatment and course of papillary thyroid a. 7 Clin Endocrinol Metab 1990;1:414-24.

Gould E, Watzak L, Chamizo W, Saavedra JA. Nuclear grooves in cytologic preparations: a study of the utility of this feature in the diagnosis of papillary carcinoma. Acta Cytol 1989;33:16-20.
3 Klinck GH, Winship T. Psammoma bodies and thyroid carcinoma. Cancer 1959;12:656-9.

4 Krevsky B. Tumors of the Schaffner F, Berk JE, eds, Bockus Gastroenterology. Philadelphia: WB Saunders, 1995; pp 534-57.

5 Gore RM. Esophageal cancer: clinical and pathologic features. Radiol Clin North Am 1997;35:243-63.

\title{
An unusual cause of dysphagia
}

\author{
Israel Gotsman, Paul Mogle, Michael Y Shapira
}

A 74-year-old woman was referred for evaluation of dysphagia and weight loss. She had a history of dysphagia for solid foods, which had become worse over the past year. She had lost $5 \mathrm{~kg}$ in weight but her appetite was good. She suffered from mild heartburn without pain and her bowel movements were normal. Gastroscopy showed mild gastritis with a positive culture for Helicobacter pylori. A short course of triple antibiotic therapy and omeprazole was prescribed. Follow-up endoscopy appeared normal. Her heartburn improved but the dysphagia persisted.

She had had a myocardial infarction 4 years earlier, complicated by acute mitral regurgitation due to papillary muscle rupture. This was repaired by urgent surgery. A post-operative echocardiogram showed slight left ventricular enlargement with decreased global function and moderate mitral regurgitation. She was treated with frusemide and digoxin and had minimal complaints of heart failure. She also suffered from type II diabetes and hypertension controlled by atenolol.

Physical examination revealed a thin woman with normal vital signs. The thyroid and lymph nodes were not enlarged. Heart sounds were normal with a $3 / 6$ blowing systolic murmur at the apex radiating to the axilla. Breath sounds were reduced at the base of the right lung. The abdomen was mildly distended but not tender. The liver and spleen were not enlarged, peristalsis was normal. There was mild oedema of both legs. Peripheral pulses were present. Laboratory studies showed mild elevation of alkaline phosphatase and $\gamma$-glutamyl transpeptidase. Albumin and cholesterol levels were normal. Haemoglobin was $14.5 \mathrm{~g} / \mathrm{dl}$. Electrocardiogram showed sinus rhythm with a non-specific intraventricular block. Chest X-ray (figure 1) demonstrated an enlarged cardiac silhouette with a small right pleural effusion.

Hadassah University Hospital, Hebrew University-Hadassah Medical School, PO Box 12000, Jerusalem, Israel 91120

Division of Medicine

I Gotsman

M Y Shapira

Department of

Radiology

P Mogle

Accepted 17 March 1999

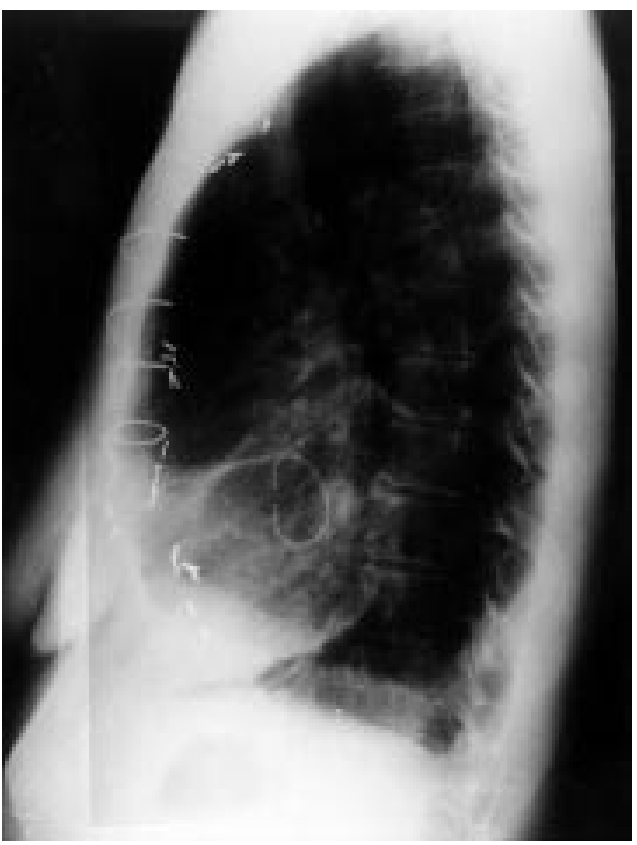

Questions

1 What is evident on the lateral chest X-ray that may explain the dysphagia?

2 What further examinations should be made in order to make a diagnosis? 


\section{Answers}

QUESTION 1

The lateral X-ray (figure 1) shows an enlarged left atrium, consistent with mitral valve disease. An enlarged left atrium can cause dysphagia to solids due to external compression of the oesophagus. This rare cause of dysphagia is known as cardiovascular dysphagia.

QUESTION 2

The patient's predominant complaint was dysphagia to solids. This usually implies mechanical obstruction of the oesophagus. Intrinsic lesions that obstruct the oesophagus include peptic stricture, lower oesophageal (Schatzki's) ring, or oesophageal carcinoma. Extrinsic lesions include vascular abnormalities, mediastinal abnormalities or cervical osteoarthritis. The first step in the diagnosis is to demonstrate the anatomy of the oesophagus by barium swallow. Video barium swallow showed normal movement of the oropharyngeal muscles and normal peristalsis of the oesophagus. A pulsatile bulge was noted in the distal posterior oesophagus consistent with external pressure from an enlarged left atrium (figure 2). No reflux was apparent. A computed tomography (CT) scan of the chest revealed an enlarged left atrium compressing the oesophagus. A space-occupying lesion was not evident. A manometric study of the oesophagus, performed to complete the diagnosis and exclude oesophageal dysmotility due to diabetes, revealed normal peristaltic pressures in the oesophagus. A transthoracic echocardiogram showed an enlarged left ventricle with moderate global dysfunction and severe mitral regurgitation. The left atrium was enlarged.

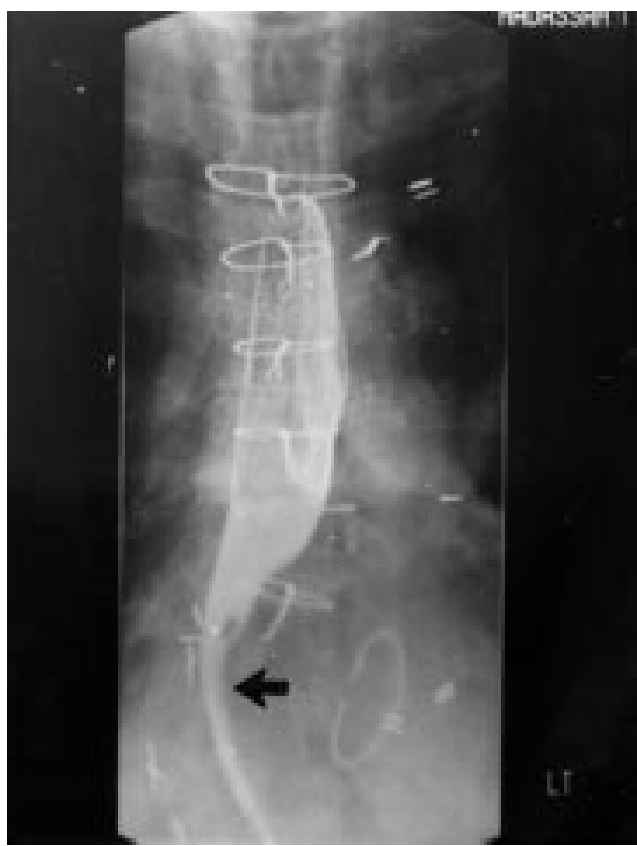

Figure 2 Barium swallow of the oesophagus showing external compression of the distal posterior oesophagus from an enlarged, left atrium (arrow). The repaired annulus of the mitral valve can be seen due to a ring placed there
The patient was treated for mitral regurgitation with after-load reduction and diuretics. She improved, the dysphagia gradually subsided, and she gained weight. The final diagnosis was cardiovascular dysphagia.

\section{Discussion}

Cardiovascular dysphagia is an uncommon clinical entity that is often unrecognised (box 1). ${ }^{1-4}$ The left atrium is a mid-line posterior chamber of the heart, in front of the oesophagus. Left atrial enlargement causes dysphagia by external compression of the oesophagus. A manometric study of left atrial enlargement ${ }^{5}$ showed that mechanical compression causes a localised high pressure zone in the oesophagus at the level of atrium with pressure oscillations corresponding to the electrocardiogram.

Other suggested mechanisms for cardiovascular dysphagia include deranged peristalsis due to local ischaemia of the oesophageal mucosa and nerve plexus caused by the elevated external pressure (which was not evident in our case). Prolonged exposure of the distal oesophagus to a high external pressure may cause proximal oesophageal muscle fatigue and dysphagia.

This patient presented with dysphagia due to mechanical compression of the oesophagus by an enlarged left atrium from mitral incompetence. Reduction of mitral incompetence by afterload reduction ameliorated the dysphagia. Cardiovascular dysphagia is uncommon, but should be suspected in patients with an enlarged left atrium.

\section{Causes of cardiovascular dysphagia}

- left atrial enlargement

- aberrant left or right subclavian artery

- tortuous atherosclerotic aorta

- thoracic aortic aneurysm

\section{Box 1}

\section{Cardiovascular dysphagia (left atrial} enlargement)

- occurs in rheumatic heart patients with mitral valve disease and enlarged left atrium

- is caused by luminal obstruction due to oesophageal compression by an enlarged left atrium

Diagnosis:

- barium swallow: posterior displaced oesophagus, a smoothly compressed oesophageal lumen, delayed passage of barium

- endoscopy: pulsatile bulging mass in the mid-oesophagus, normal mucosa

- oesophageal manometry: elevated mid-esophageal base line pressure with superimposed cyclic pressure waves simultaneous with the ECG 
Final diagnosis

Cardiovascular dysphagia.

Cappell MS. Manometric findings in dysphagia secondary to left atrial dilatation. Dig Dis Sci 1991;36:693-8.

2 McNally PR, Rak KM. Dysphagia lusoria caused by persistent right aortic arch with aberrant left subclavian artery and diverticulum of Kommerell. Dig Dis Sci 1992;37:144-9.

3 Berenzweig H, Baue AE, McCallum RW. Dysphagia lusoria: Berenzweig $\mathrm{H}$, Baue AE, McCallum RW. Dysphagia lusoria:
report of a case and review of the diagnostic and surgical approach. Dig Dis Sci 1980;25:630-6.
Keywords: cardiovascular dysphagia; mitral valve disease; weight loss

4 Mittal RK, Siskind BN, Hongo M, et al. Dysphagia aortica: clinical, radiological and manometric findings. Dig Dis Sci 1986;31:379-84.

5 Cappell MS. Endoscopic, radiographic and manometric findings associated with cardiovascular dysphagia. Dig Dis Sci 1995;40:166-76.

\title{
Lessons from a case of tetanus in an elderly woman
}

\author{
J Kwan, S Lim, S C Allen
}

A healthy 84-year-old Caucasian woman fell whilst gardening at home and lacerated her right leg. The wound was deep and heavily contaminated with soil and gravel. She attended an Accident and Emergency (A\&E) department for treatment and the wound was cleaned and dressed. She had never received tetanus vaccination in the past and at the $\mathrm{A} \& \mathrm{E}$ department she was given one dose of tetanus toxoid. She was sent home without any follow up. Over the next week she became increasingly immobile and complained of dysphagia, stiff neck and breathlessness. Her general practitioner admitted her into a nursing home with a presumed diagnosis of stroke. Her condition deteriorated over the next week and she was admitted into hospital with a provisional diagnosis of septicaemia from her original leg wound.

On admission she was dehydrated, hypoxic, hypertensive $(230 / 125 \mathrm{mmHg})$ and tachycardic. She had generalised muscular spasms including trismus and neck stiffness as well as hyperexcitability to touch and noise. Spirometry showed a restrictive lung defect. Initial laboratory results were as follows: leucocytes $15.5 \times 10^{9} / 1$, platelets $653 \times 10^{9} / 1$, urea $15.4 \mathrm{mmol} / 1$, creatinine 227 $\mu \mathrm{mol} / 1$ and corrected calcium $2.48 \mathrm{mmol} / 1$. A diagnosis of tetanus was made and she was transferred to the Intensive Care Unit where she was managed according to recommended guidelines for the treatment of tetanus. Her pulse and blood pressure observations were monitored closely (table).

Table Blood pressure and pulse observations on day 7

Royal Bournemouth \& Christchurch Hospitals NHS Trust, Castle Lane East, Bournemouth BH7 7DW, Dorset, UK J Kwan

S Lim

S C Allen

Correspondence to Dr Joseph S K Kwan, Research Fellow,

Christchurch Hospital, Fairmile Road, Christchurch BH23 2JX, Dorset, UK

Accepted 18 February 1999

\begin{tabular}{lll}
\hline Time & Blood pressure $(\mathrm{mmHg})$ & Pulse (beats/min) \\
\hline $01: 00$ & $122 / 50$ & 80 \\
$04: 00$ & $90 / 40$ & 70 \\
$07: 00$ & $125 / 60$ & 80 \\
$12: 00$ & $150 / 50$ & 100 \\
$20: 00$ & $160 / 70$ & 120 \\
$22: 00$ & $130 / 70$ & 88 \\
\hline
\end{tabular}

\section{Questions}

1 Comment on the original A\&E department management of this patient.

2 What caused the abnormally unstable blood pressure and pulse?

3 What are the most important aspects of the management of tetanus?

4 What is the differential diagnosis and how may the initial diagnosis be confirmed? 